\title{
Proceso de selección flexible por competencias aplicado al puesto de Dircom
}

\author{
Rita Jácome López | rita.jacome@iecoinstitute.org \\ Instituto para la Ética en la Comunicación y en las Organizaciones \\ Lourdes Canós Darós | loucada@omp.upv.es \\ Universitat Politècnica de València \\ Alexis Jacobo Boñón Gomis | albolago@upvnet.upv.es \\ Universitat Politècnica de València
}

Recibido en: 19 de mayo de 2016

Aceptado en: 1 de septiembre de 2016

\section{RESUMEN}

El puesto de Director de Comunicación es relevante para la reputación de una empresa, pues influye a través de su propia imagen y reputación personal; por tanto, la selección de este directivo debe ser cuidadosa. En este trabajo proponemos un proceso de selección flexible basado en la lógica borrosa, para ajustar las competencias de los candidatos al puesto y dar apoyo a la toma de decisiones. Presentamos dos técnicas: una en la que se selecciona al mejor solicitante y otra en la que se compara a los candidatos con un ideal construido con información facilitada por directores españoles.

Palabras clave: conjuntos borrosos, impression management, reputación corporativa, reputación personal, selección de personal

\section{ABSTRACT}

The Communication Manager position is relevant to the reputation of an organization, because it influences through its own personal image and reputation; therefore, the selection of this manager has to be painstaking. In this paper we propose a flexible selection process based on fuzzy logic, to fit the skills of candidates for the job and to support decision making. We present two techniques: one that selects the best applicant and another one that compares candidates with an ideal constructed with information provided by Spanish managers.

Keywords: fuzzy sets, impression management, corporate reputation, personal reputation, personnel selection 
Este trabajo presenta un modelo borroso flexible de selección de personas por competencias, elaborado según las variables presentadas en el modelo relacional de reputación, desde la teoría de la impression management (IM) o gestión de las impresiones de Jácome (2015). Dicho modelo sienta sus bases en la visión integradora y relacional de las tres dimensiones constitutivas de la IM, equivalentes a los tres elementos clave de la reputación, tanto personal como organizacional (identidad personal, identidad social e identidad profesional o corporativa). Esta premisa, unida a la noción de que la reputación puede ser transferida del individuo a la organización y viceversa (Schweizer \& Wijnberg, 1999), ha permitido explicitar en qué medida la IM puede ser considerada como antecedente o generador de reputación personal (RP) y a su vez cómo la reputación a nivel corporativo (RC) podría ser considerada como beneficio o resultado de una RP favorable.

El objetivo de este estudio es aplicar dicho modelo conceptual a un modelo flexible de selección de directores de comunicación o Dircom en España. Estos profesionales, situados en la línea de la alta dirección de las empresas en las que trabajan, por su desempeño y las responsabilidades que ostentan, suelen formar parte de los consejos de administración. Se trata, por tanto, de trabajadores que juegan un papel relevante en la toma de decisiones y en el diseño de la estrategia corporativa de la organización a la que representan. Dentro del marco institucional en el que desarrollan su actividad, su representatividad se extiende al hecho de ser responsables de velar por la imagen del máximo ejecutivo de la compañía -su presidente- así como de tratar de controlar la proyección pública de la organización ante los grupos de interés, internos y externos, incluida la opinión pública.

El término anglosajón IM hace referencia al proceso por el que las personas, de forma más o menos consciente, tratan de controlar la imagen que proyectan al exterior con el objetivo de causar en los demás unas determinadas impresiones (Leary \& Kowalski, 1990; Schlenker, 1980). La perspectiva de la gestión de las impresiones que propone este trabajo es una visión integradora de la teoría de la IM, que estaría fundamentada sobre la base de la integridad del 
comportamiento (behavioral integrity) o la adecuación entre lo que una persona dice y lo que hace (Kacmar \& Tucker, 2014; Palanski, Kahai, \& Yammarino, 2011; Simons, 2002). El proceso de la IM abarca una serie de técnicas y modos de obrar que afectan a los distintos ámbitos del comportamiento humano. Se trataría de gestionar una serie de técnicas de comunicación verbal - autopromoción, ejemplaridad, súplica o petición- para ayudar a definir qué se dice y cómo se dice, así como de comunicación no verbal - apariencia física, expresiones faciales, posturas e incluso el mobiliario y la decoración de que se rodea una persona- (Rosenfeld, Giacalone, \& Riordan, 1995). Todo ello con el objetivo de proyectar una determinada imagen de la dimensión de la propia identidad (personal, social o profesional), para ser percibidos por los demás de forma favorable.

La distorsión de las propias impresiones sería más susceptible de ser lograda en orden a conseguir transmitir una dimensión concreta de la identidad no acorde con la propia personalidad a nivel operativo y táctico - a corto y medio plazo-; sin embargo, es en el largo plazo donde se plantea la necesidad de poner en relación la IM con la gestión de la reputación. Una visión integradora de las tres dimensiones de la identidad en los diferentes contextos o entornos (ético, social y organizativo) llevaría a la idea de la consistencia en el comportamiento (Jácome, Bañón, Guillén, \& Canós, 2013). Contar con una buena reputación implicaría que la persona pueda ser estimada, valorada, respetada y admirada en todos los contextos y de manera sostenida en el tiempo; es por esto que se han considerado las competencias identificadas de las escalas que miden las distintas técnicas de IM como antecedentes de la RP. Esta comienza a construirse a partir de la formación de unas percepciones iniciales sobre las características personales del individuo y sus comportamientos que, si se hacen consistentes en el tiempo, dejan una huella por la que los demás reconocen y hacen evaluaciones morales y éticas de la persona en término de buena o mala (Anderson \& Shirako, 2008; Bromley, 2001; Foste \& Botero, 2012). Estas competencias o comportamientos observables y habituales constitutivos de la RP, según se han identificado 
en el modelo relacional de reputación de Jácome (2015) son la confianza y el liderazgo. Asimismo, la literatura ha utilizado variedad de escalas para medir los distintos atributos de la IM, lo que ha permitido la identificación de una serie de competencias de dimensión ética, afectiva y técnica, según las tres dimensiones de la confianza (Mayer, Davis, \& Schoorman, 1995) y el liderazgo (Guillén \& González, 2001), que se han considerado como antecedentes de la RP (Figura 1).

\section{Dimensión ética

Ejemplaridad \\ Asumir el error}

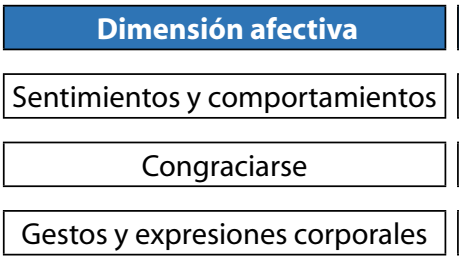

Gestos y expresiones corporales
Dimensión técnica

\begin{tabular}{|c|}
\hline Autopromoción \\
\hline Desempeño laboral \\
\hline Aspecto físico \\
\hline Comunicar con la apariencia \\
\hline Cualificación \\
\hline Capital humano \\
\hline
\end{tabular}

Figura 1. Competencias de la impression management según las dimensiones de la confianza y el liderazgo. Fuente: Elaboración propia a partir del modelo relacional de reputación personal de Jácome (2015).

Por otro lado, la definición de RG que se ha adoptado en este trabajo de acuerdo con la escuela relacional de reputación sería "la suma relativamente estable de las percepciones de los grupos de interés internos y externos para lograr juicios evaluativos y como resultado, suscitar comportamientos de apoyo u oposición a una organización" (Jácome, 2015, p. 109). La imagen (lo que los grupos de interés externos piensan que es la organización), la identidad (lo que los grupos de interés internos creen que la organización es actualmente: cultura corporativa) y la identidad deseada (lo que la organización comunica que es: misión, visión y valores) serían los elementos clave que, sumados, contribuirían a construir RC (Chun, 2005). Se trata de un juicio evaluativo en términos comparativos de bueno, correcto, positivo o malo, incorrecto, negativo, basado en un conjunto de percepciones y en la experiencia, que se forma a lo 
largo del tiempo siendo un fenómeno más o menos estable, duradero y focalizado en lo que la persona hace y en cómo se comporta (Brown, Dacin, Pratt, \& Whetten, 2006; Chun, 2005; Fombrun, 1996; Scott \& Lane, 2000; Walker, 2010). La variedad de escalas que se han utilizado en la literatura para medir los distintos atributos de la RC han permitido la identificación de una serie de competencias de dimensión ética, afectiva y técnica que, en este trabajo, se han considerado como consecuentes de la RP (Figura 2).

\begin{tabular}{|c|c|c|}
\hline Dimensión ética & Dimensión afectiva & Dimensión técnica \\
\hline Ética & Orientación al cliente & Competencia \\
\hline Honestidad & Credibilidad & Autonomía \\
\hline Responsabilidad social & Entusiasmo & Prestigio \\
\hline \multirow[t]{7}{*}{ Integridad } & Factores motivadores & Habilidades directivas \\
\hline & Comunicación emocional & Promoción \\
\hline & Cultura corporativa & Buena apariencia \\
\hline & & Análisis del entorno \\
\hline & & Calidad \\
\hline & & Solvencia financiera \\
\hline & & Estrategia corporativa \\
\hline
\end{tabular}

Figura 2. Competencias de reputación corporativa según las dimensiones de la confianza y el liderazgo. Fuente: Elaboración propia a partir del modelo relacional de reputación personal de Jácome (2015).

Dos de las principales aportaciones de la IM a la gestión de las organizaciones se encuentran en las políticas de selección de personas y en el ajuste del candidato al puesto y a la organización a través de la evaluación (Cable \& Judge, 1997; Chen \& Lin, 2014; Higgins \& Judge, 2004; Kristof-Brown, 2000). En este contexto, muchos trabajos enmarcados en el ámbito de los recursos humanos se han centrado en el análisis de dos técnicas, congraciarse y autopromoción, ambas destinadas a conectar con el interlocutor que puede ser el 
responsable de un proceso de selección, si el individuo pretende incorporarse a una determinada organización, o bien un superior, en el caso de tratarse de una evaluación de personal (Bolino, Klotz, \& Daniels, 2014; Ellis, West, Ryan, \& DeShon, 2002; Jones \& Pittman, 1982; Proost, Schreurs, Witte, \& Derous, 2010; Wayne \& Kacmar, 1991).

El proceso de selección de personas puede ser complicado por el tipo de vacante que se desea cubrir o por la cantidad de candidatos que se presentan para el trabajo. Además, pueden entrar en juego varios criterios, con lo que los responsables del proceso han de ejecutar una agregación de información eficiente y flexible para tratar los datos con precisión sin perder la calidad de la información (Larsen, 2002). En este trabajo se define la selección de personal como un proceso en el que un responsable elige a una o varias personas en función de su ajuste a las características de un puesto de trabajo vacante (Canós, 2005; Valle Cabrera, 2003). Obviamente, el resultado de este proceso será una ordenación de los candidatos en función del criterio que se haya utilizado para medir su ajuste al puesto de trabajo vacante.

En este contexto, podemos considerar la aplicación de técnicas flexibles de selección de personas. La teoría en la que se ha basado la propuesta es la desarrollada en los 60 sobre subconjuntos borrosos (Zadeh, 1965). Aunque existen distintas maneras de incluir la incertidumbre en el formalismo de un modelo de decisión, se ha optado por esta teoría por varios motivos (Canós \& Liern, 2008; Liern \& Canós, 2003):

- Evita que la rigidez de las limitaciones del modelo hagan que resulte poco factible o sin sentido.

- Impide ignorar soluciones del problema que resultarían provechosas, pero que no se pueden obtener con una formalización matemática tradicional.

- Incorpora al modelo la subjetividad del decisor y la incertidumbre del proceso por su propio planteamiento, lo que resulta ideal para la toma de decisiones sobre recursos humanos dada su casuística. 
La teoría de conjuntos borrosos se basa en la sustitución de los conjuntos tradicionales (en los que un elemento puede pertenecer o no pertenecer) por funciones de pertenencia. Las funciones de pertenencia son aplicaciones de un conjunto referencial dado $\mathrm{X}$ en el intervalo [0,1] (Goguen, 1967, 1969; Zadeh, 1965). Si $\mu$ Ã es la función de pertenencia del subconjunto borroso A entonces $\mu$ A (x) se interpreta como el grado de pertenencia del elemento $\mathrm{x}$ al conjunto A (Kaufmann \& Gil Aluja, 1987). Si el grado de pertenencia de un elemento es cero, no existe pertenencia al conjunto; si es uno, pertenece por completo como en los conjuntos tradicionales (booleanos); si la pertenencia es un número intermedio existe una incertidumbre que será interpretada de diversos modos según cada aplicación (Liern \& Canós, 2003).

\section{Identificación y definición de competencias más significativas para el desarrollo del puesto de Dircom}

Para el desarrollo del puesto de Dircom se han considerado un total de 12 competencias identificadas a partir de la simplificación del modelo relacional de RP propuesto por Jácome (2015). Se han incluido cuatro variables adicionales: capital humano, credibilidad, integridad y prestigio porque, a pesar de no encontrase en dicha simplificación del modelo relacional de RP del Dircom español, sí que se encuentran en el modelo general; por tanto, se ha decidido añadirlas para su evaluación por considerarlas representativas a la hora de ser valoradas en un proceso de selección de personas para un puesto de Dircom. A continuación se ofrece una breve descripción de cada una de ellas:

1. Capital humano. Constituye la parte de los activos intelectuales conocidos como valores intangibles (Fombrun \& Low, 2011; Zinko, Ferris, Humphrey, Meyer, \& Aime, 2012). Según Fombrun y Low (2011), el valor de una organización no solamente reside en el capital financiero y en los activos físicos sino también en el capital humano. En este sentido, la formación y la experiencia de los trabajadores reconoci- 
dos como tales constituyen un antecedente de dimensión técnica de la RP.

2. Competencia. Consiste en poseer la capacidad para desarrollar una actividad específica (Helm, 2005). Se trata de uno de los cinco factores de la personalidad humana establecidos por Aaker (1997) y que posteriormente algunos autores, basándose en la metáfora de la personificación, han utilizado para atribuírselos a una marca u organización (Davies, Chun, da Silva, \& Roper, 2004). Los conocimientos, las competencias o aptitudes y las habilidades se tienen más en cuenta cuando se pretende establecer la idoneidad de un candidato al puesto de trabajo en procesos de recursos humanos (Kristof-Brown, 2000).

3. Confianza. Es "la decisión de una persona de hacerse vulnerable ante una acción de otro. Esta decisión está basada en la suposición de que la persona en la que se confía hará bien esa acción sin que se le controle" (Mayer et al., 1995, p. 712). En este trabajo se ha considerado esta variable como uno de los dos integrantes de la RP.

4. Congraciarse. Es la capacidad de conseguir la benevolencia, la aprobación o el afecto de alguien (Kumar \& Beyerlein, 1991), así como la empatía y cierta atracción interpersonal (Ellis et al., 2002). En el modelo propuesto en este trabajo de investigación se trata de un antecedente de la RP de dimensión afectiva.

5. Credibilidad. Es uno de los factores principales que determinan el poder de la comunicación persuasiva, ya que tiene que ver con la autenticidad del carácter del orador para inspirar credibilidad en su audiencia (logos). Como consecuente de la RP de dimensión afectiva, la credibilidad de una organización reside en la creencia por parte de los consumidores de la intención de la compañía en un determinado momento, de que la organización puede diseñar y ofrecer productos y servicios que satisfagan sus deseos y necesidades (Keller, 1993). 
6. Desempeño laboral. Forma en que los trabajadores desempeñan bien su trabajo. Afecta a la competencia del empleado para desempeñar un determinado puesto (Foste \& Botero, 2012). Se evalúa mediante revisiones del rendimiento laboral. En este trabajo es una competencia considerada como antecedente de la RP de dimensión técnica.

7. Ética. "La confianza en la expectativa de que una persona o grupo actuará de una manera moralmente responsable al interactuar con otras en una operación o intercambio" (Adams, Highhouse, \& Zickar, 2010, p. 40). Esta definición se ha tomado de los autores que han desarrollado la escala de la desconfianza para medir el impacto de esta competencia en la reputación a nivel organizacional. Además de esperar que una de las partes actúe según lo acordado, para estos autores la actuación ética se refiere también a las expectativas del conjunto social que espera que una organización se rija por un proceso de toma de decisiones morales. En este trabajo se ha considerado esta competencia como una variable de la RC de dimensión ética, consecuente de la RP.

8. Honestidad. Es una cualidad intangible que, aplicada a una marca, se identifica con la honradez, la lealtad, la sinceridad y la justicia, puesto que la marca no es solo un nombre, un logo, un símbolo o un nombre comercial (Afzal, Khan, ur Rehman, Ali, \& Wajahat, 2010). La honestidad es parte de la sinceridad, uno de los atributos de las marcas que la autora Jennifeer L. Aaker mide con su modelo de los cinco factores. Esta escala está basada en la metáfora de la personificación consistente en asociar características de la personalidad humana a una marca (Aaker, 1997, p. 352). Se trata de una competencia de dimensión ética consecuente de la RC.

9. Integridad. Es la consistencia interna del carácter, lo que hace a una persona recta e intachable en su conducta (Afzal et al., 2010). Al igual que la competencia anterior, se trata también de una cualidad intangible relacionada con la marca, una competencia de dimensión ética consecuente de la RC. 
10. Liderazgo. Es una reacción establecida por el sujeto actuante desde la libertad y sin obligación externa de seguir a otra persona, el líder. "El liderazgo va más allá del poder formal, implica un continuo intercambio de influencia y de aceptación gratuita" (Guillén \& González, 2001, p. 176). En este trabajo se ha considerado esta variable como uno de los dos integrantes de la RP junto con la confianza. Se ha tomado como referencia la definición de los autores Guillén y González (2001) ya que, como se ha explicado anteriormente, se han adoptado las tres dimensiones del liderazgo que ellos establecen a las dimensiones de las competencias identificadas en nuestro modelo de reputación.

11. Prestigio. Consiste en ser considerado en alta estima por aquellas personas que observan al individuo desde fuera de la organización. El prestigio de un individuo depende, entre otros, del prestigio de aquellos con los que socializa y le aceptan como un igual (Sung \& Yang, 2008). La definición de esta competencia se basa en la idea de que las personas muestran admiración por las organizaciones fundamentalmente a través del respeto y el asombro. Es decir, una entidad tiene prestigio si se admira, porque es importante por su honor e integridad (Highhouse, Brooks, \& Gregarus, 2009). En este trabajo la variable del prestigio se ha considerado como un consecuente de la RC de dimensión técnica.

12. Responsabilidad social. Se refiere a las expectativas que la organización genera en sus trabajadores en cuanto a la igualdad en el trato y la adhesión de la compañía a las reglas establecidas (Walsh \& Beatty, 2007). En la escala de reputación corporativa basada en el cliente desarrollada por dichos autores, los cuatro ítems que definen la categoría de responsabilidad social y medioambiental son: "Parece que pone empeño en crear nuevos puestos de trabajo; estaría dispuesto a reducir sus beneficios por asegurar un entorno ambiental limpio; parece que es medioambientalmente responsable; se ofrece para apoyar buenas causas" (Walsh \& Beatty, 2007, p. 135). 
Siguiendo lo establecido en el modelo relacional de RP del Dircom español (Jácome, 2015), estas 12 competencias se agrupan del siguiente modo (Figura 3):

- Pertenecen a la categoría de IM como antecedentes a la RP: 1) Capital humano; 4) Congraciarse; 6) Desempeño laboral.

- Pertenecen a la categoría de RC como consecuentes de la RP: 2) Competencia; 5) Credibilidad; 7) Ética; 8) Honestidad; 9) Integridad; 11) Prestigio; 12) Responsabilidad social.

- Pertenecen a la categoría de RP como integrantes del constructo: 3) Confianza; 10) Liderazgo.

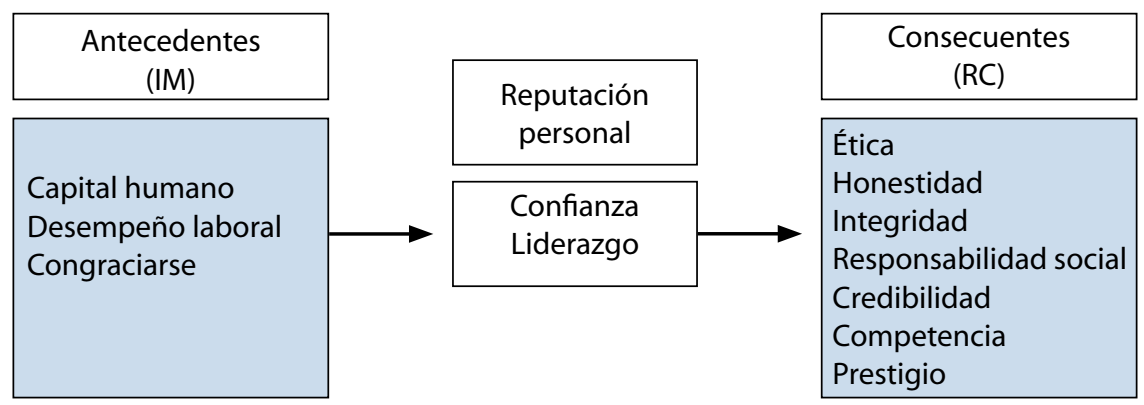

Figura 3. Agrupación de competencias según el modelo de antecedentes y consecuentes de RP de Jácome (2015). Fuente: Elaboración propia a partir de Jácome (2015).

\section{Aplicación de técnicas flexibles de selección de personas para un puesto de Dircom}

Nuestra propuesta parte del supuesto de que existe un puesto vacante de Dircom en una organización de cualquier tipo y que los cinco candidatos finalistas han obtenido los siguientes resultados en la evaluación de sus competencias (Tabla 1). 
Tabla 1

Evaluación de las competencias de los candidatos

\begin{tabular}{|c|c|c|c|c|c|c|c|c|c|c|}
\hline \multirow[b]{3}{*}{ Capital humano } & \multicolumn{2}{|c|}{ Candidato 1} & \multicolumn{2}{|c|}{ Candidato 2} & \multicolumn{2}{|c|}{ Candidato 3} & \multicolumn{4}{|c|}{ Candidato 4 Candidato 5} \\
\hline & Mín. & Máx. & Mín. & Máx. & Mín. & Máx. & Mín. & Máx. & Mín. & Máx. \\
\hline & 5 & 7 & 8 & 9 & 5 & 6 & 2 & 5 & 5 & 7 \\
\hline Competencia & 3 & 6 & 7 & 10 & 6 & 8 & 9 & 10 & 2 & 6 \\
\hline Confianza & 6 & 7 & 9 & 9 & 5 & 8 & 4 & 6 & 9 & 10 \\
\hline Congraciarse & 5 & 8 & 8 & 9 & 7 & 10 & 3 & 5 & 5 & 5 \\
\hline Credibilidad & 2 & 3 & 8 & 9 & 9 & 9 & 7 & 7 & 7 & 7 \\
\hline $\begin{array}{l}\text { Desempeño } \\
\text { laboral }\end{array}$ & 4 & 5 & 10 & 10 & 4 & 7 & 7 & 10 & 6 & 9 \\
\hline Ética & 1 & 2 & 9 & 10 & 6 & 10 & 5 & 6 & 3 & 4 \\
\hline Honestidad & 1 & 3 & 8 & 10 & 8 & 9 & 6 & 9 & 6 & 8 \\
\hline Integridad & 2 & 5 & 9 & 10 & 5 & 7 & 5 & 7 & 6 & 10 \\
\hline Liderazgo & 4 & 6 & 7 & 9 & 6 & 9 & 3 & 7 & 8 & 10 \\
\hline Prestigio & 2 & 4 & 7 & 9 & 4 & 9 & 7 & 8 & 7 & 8 \\
\hline $\begin{array}{l}\text { Responsabilidad } \\
\text { social }\end{array}$ & 2 & 2 & 6 & 9 & 6 & 7 & 7 & 9 & 7 & 9 \\
\hline
\end{tabular}

Fuente: Elaboración propia a partir de Jácome (2015).

Los candidatos han sido evaluados usando intervalos para flexibilizar el proceso. Es habitual valorar a una persona en algo como "más o menos bueno" o "regular", y una forma de trasladar estas afirmaciones a la formalización de los modelos de decisión es a través de los intervalos.

\section{Sin candidato ideal}

La primera idea que puede tener el responsable de la selección de personal es la de maximizar las competencias que puedan tener los candidatos para ordenarlos y conocer quién es el mejor en términos globales. En realidad, se trata de una comparación con el máximo posible. Esta es una opción válida, sobre todo si se da el caso de que, por ejemplo, no existe un ideal, porque no es posible definirlo en industrias de reciente creación (Fullér, Canós, \& Canós, 2012); no obstante, su aplicación en situaciones normales no está exenta de críticas, pues una persona que empieza a trabajar 
en un puesto para el que no se ajustan sus competencias puede resultar frustrada y querer cambiar de trabajo en cuanto tenga una oportunidad.

En este caso, para simplificar el proceso, se ha buscado un representante de cada intervalo, considerando la media entre el mínimo y el máximo. Se podría haber elegido otro punto, por ejemplo, con una fórmula más optimista, pero se entiende que esta representación es adecuada.

Puesto que en realidad se está comparando con el máximo posible, hay que calcular una media para cada candidato (su valoración para la ordenación final) midiendo la distancia de cada una de las competencias con el máximo, que en este caso, toma el valor de 10 . Entonces, se aplica la siguiente expresión (Fullér et al., 2012):

$$
\frac{1}{R} \sum_{i=1}^{R}\left(1-b_{c i}^{j}\right)
$$

Donde R es el número total de competencias y b es la valoración de cada una de las competencias para cada uno de los candidatos. Siguiendo el ejemplo propuesto, el resultado final después de aplicar este método es: Candidato 1 = 6,04; Candidato $2=1,29$; Candidato $3=3,41$; Candidato $4=3,75$; Candidato $5=3,16$.

A continuación se ordena a los candidatos de menor a mayor resultado, pues lo que se está midiendo es la distancia con el máximo (el que esté más cercano al máximo es el mejor). Los resultados muestran que el Candidato 2 sería el primero en ocupar el puesto, si se sigue esta herramienta de decisión. Este resultado tiene sentido, pues ya se intuía a priori por las altas valoraciones en las competencias que, de forma general, tenía el Candidato 2 en la presentación de los datos.

\section{Con candidato ideal}

Otras técnicas de ordenación que el responsable del proceso de selección puede utilizar son las que se basan en la comparación con un candidato ideal, construido con la opinión de expertos (Canós $\&$ Liern, 2004). En este caso, los expertos en los que se fundamenta el ideal que se va a presentar han sido ocho directores de comuni- 
cación de empresas internacionales sitas en Madrid, Barcelona y Valencia (Tabla 2). El ideal es la media de cada competencia y se ha calculado utilizando los puntos medios de las valoraciones mínimas y máximas que cada experto ha dado a cada competencia.

Con estos datos, se analiza el ajuste entre los datos procedentes de los candidatos y el ideal propuesto por los directores de comunicación utilizando el índice de adecuación, que se define formalmente como:

$$
\mu_{\tilde{I}^{\phi}}\left(\tilde{P}_{j}^{\phi}\right)=\frac{1}{n} \sum_{\mathrm{i}=1}^{n} \mu_{\tilde{I}^{\phi}}^{x_{i}}\left(\tilde{P}_{j}^{\phi}\right), \operatorname{donde} \mu_{\mathrm{I}^{\phi}}^{\mathrm{x}_{\mathrm{i}}}\left(\mathrm{P}_{\mathrm{j}}^{\phi}\right)=\frac{\operatorname{long}\left(\left[\mathrm{b}_{\mathrm{x}_{\mathrm{i}}}^{1}, \mathrm{~b}_{\mathrm{x}_{\mathrm{i}}}^{2}\right] \cap\left[\mathrm{a}_{\mathrm{x}_{\mathrm{i}}}^{1}, \mathrm{a}_{\mathrm{x}_{\mathrm{i}}}^{2}\right]\right)}{\operatorname{long}\left(\left[\mathrm{b}_{\mathrm{x}_{\mathrm{i}}}^{1}, \mathrm{~b}_{\mathrm{x}_{\mathrm{i}}}^{2}\right] \cup\left[\mathrm{a}_{\mathrm{x}_{\mathrm{i}}}^{1}, \mathrm{a}_{\mathrm{x}_{\mathrm{i}}}^{2}\right]\right)} .
$$

Los resultados se ordenan de mayor a menor, pues el candidato más adecuado para el puesto es el que tiene una mayor similitud con el perfil ideal previamente definido: Candidato $1=5,78$; Candidato $2=8,11$; Candidato $3=9,94$; Candidato $4=9,27$; Candidato $5=8,72$. El Candidato 3 es el que tiene un mayor valor, por lo que es el más adecuado para ocupar el puesto según el coeficiente de adecuación.

Como se puede observar, utilizando la herramienta del índice de adecuación, el resultado es diferente al que podríamos haber supuesto a priori al ver las valoraciones de las competencias de los candidatos. Considerando el máximo absoluto, esto es, el candidato más cercano a la perfección en todas las competencias, el Candidato 2 era el óptimo, mientras que con esta técnica, formalizada para ajustar el puesto a la persona, queda relegado al puesto número cuatro y el Candidato 3 es el primero en la lista ordenada. La decisión final depende del responsable del proceso de selección. En este proceso, las herramientas de ayuda a la toma de decisiones presentadas en este capítulo pueden ser de utilidad. 
Proceso de SelecCión fleXible POR COMPETENCIAS APLICAdo AL PUESTO de DiRCoM

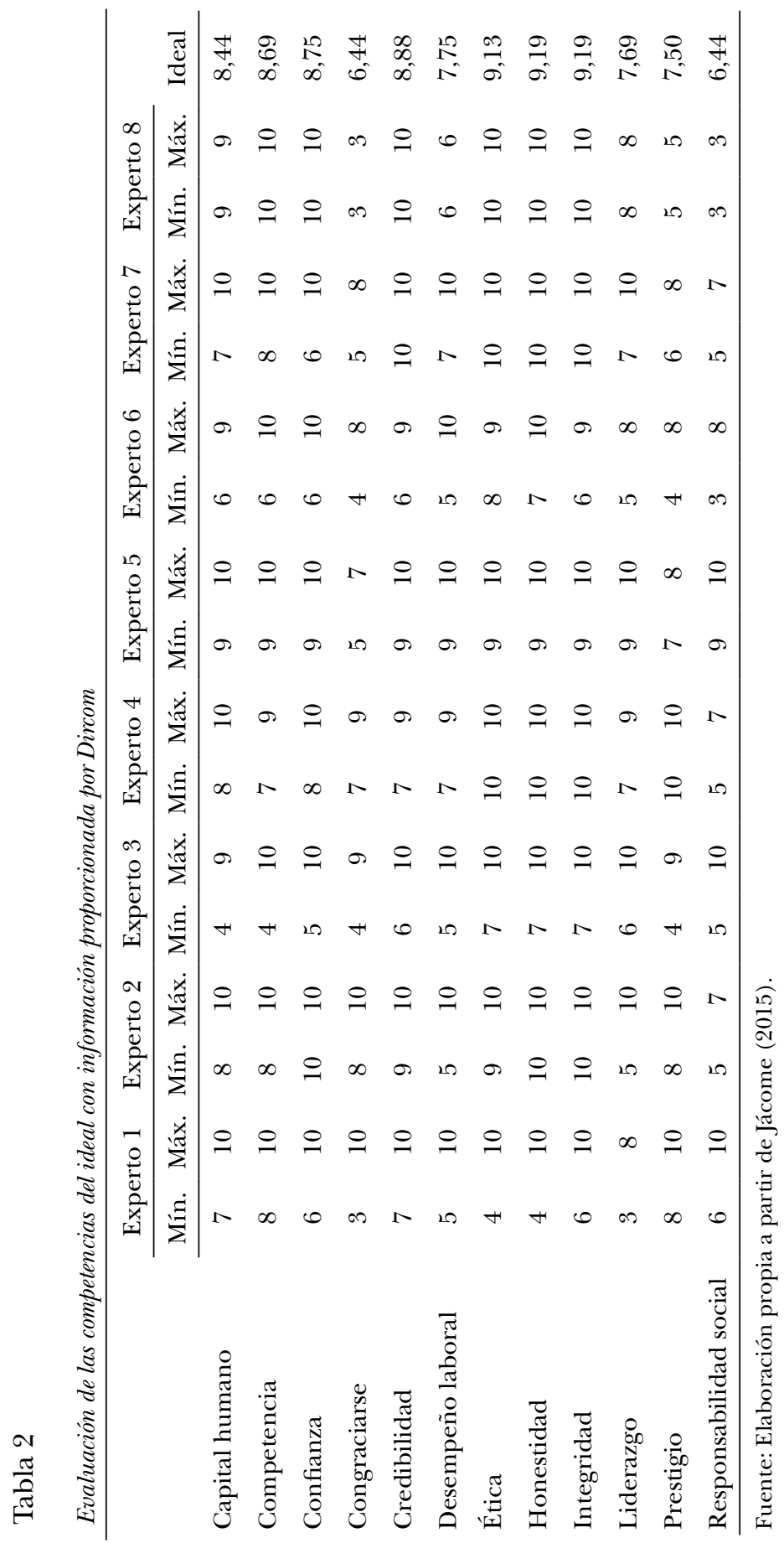




\section{Conclusiones, limitaciones y futuras líneas de investigación}

Este trabajo presenta un modelo flexible de selección de personas adaptado a la elección de un Dircom; para ello, se han usado técnicas borrosas, de forma que se ha incluido en el proceso la subjetividad inherente a la toma de decisiones sobre personas y la flexibilidad que requiere la valoración de ciertas competencias, actitudes, aptitudes, etc., en el ámbito de los recursos humanos. En la aplicación de la herramienta se han distinguido dos casos: cuando no existe un referente y cuando se puede comparar a los candidatos al puesto con un ideal.

En este orden de ideas, se proponen una serie de competencias identificadas en las escalas que miden distintas técnicas de IM como antecedente de la RP y RC como su consecuente, destiladas de la literatura. Estas competencias son: capital humano, competencia, confianza, congraciarse, credibilidad, desempeño laboral, ética, honestidad, integridad, liderazgo, prestigio y responsabilidad social. Asimismo, para corroborar estas características, se ha consultado a ocho Dircom que, además de dar su opinión sobre un posible candidato ideal para ocupar este puesto, han confirmado la importancia de estas competencias. En este sentido, los Dircom consultados han puesto en valor la creación de un candidato ideal a través de su experiencia y deseos de mejora, en la definición del puesto de trabajo.

Como principal limitación se puede citar la dificultad de encontrar un proceso real de selección de Dircom al que aplicar estos modelos. Aunque la fiabilidad de las herramientas ha sido probada en empresas reales y se ha confirmado que los resultados ayudan a la toma de decisiones del Director de Recursos Humanos (Canós, Casasús, Crespo, Lara, \& Pérez, 2011; Canós, Casasús, Lara, Liern, \& Pérez, 2008), es complicado que quede una vacante en este tipo de puesto por su especialidad y porque la mayoría de las empresas no tienen este puesto explícitamente definido. Nuestra intención es encontrar alguna oportunidad en el futuro para poder presentar resultados reales que no procedan de una simulación. 
Del mismo modo, pensamos mejorar los modelos presentados adaptándolos a otras políticas de recursos humanos relacionadas con la selección de personal, como son la evaluación del desempeño, la formación o la motivación. El objetivo final sería el diseño de una herramienta integral de gestión de recursos humanos que se realimentará con la información que se genere en los distintos procesos por los que pasa un empleado, desde el momento en que es contratado en la empresa. 


\section{Referencias}

Aaker, J. L. (1997). Dimensions of brand personality. Journal of Marketing Research, 24, 347-356.

Adams, J. E., Highhouse, S., \& Zickar, M. J. (2010). Understanding general distrust of corporations. Corporate Reputation Review, 13(1), 38-51.

Afzal, H., Khan, M. A., ur Rehman, K., Ali, I., \& Wajahat, S. (2010). Consumer's trust in the brand: Can it be built through brand reputation, brand competence and brand predictability. International Business Research, 3(1), 43-51.

Anderson, C., \& Shirako, A. (2008). Are individuals' reputations related to their history of behavior? Journal of Personality and Social Psychology, 94(2), 320-333.

Bolino, M. C., Klotz, A. C., \& Daniels, D. (2014). The impact of impression management over time. Journal of Managerial Psychology, 29(3), 266-284.

Bromley, D. B. (2001). Relationships between personal and corporate reputation. European Journal of Marketing, 35(3/4), 316-334.

Brown, T. J., Dacin, P. A., Pratt, M. G., \& Whetten, D. A. (2006). Identity, intended image, construed image, and reputation: An interdisciplinary framework and suggested terminology Academy of Management Journal, 34(2), 99-106.

Cable, D. M., \& Judge, T. A. (1997). Interviewers' perceptions of person-organization fit and organizational selection decisions. Journal of Applied Psychology, 82(4), 546-561.

Canós, L. (2005). Gestión de recursos humanos basada en la lógica borrosa. Rect@: Revista Electrónica de Comunicaciones y Trabajos de ASEPUMA, 6, 29-60.

Canós, L., Casasús, T., Crespo, E., Lara, T., \& Pérez, J. C. (2011). Personnel selection based on fuzzy methods. Revista de Matemática. Teoría y Aplicaciones, 18(1), 177-192.

Canós, L., Casasús, T., Lara, T., Liern, V., \& Pérez, J. C. (2008). Modelos flexibles de selección de personal basados en la valoración 
de competencias. Rect@: Revista Electrónica de Comunicaciones y Trabajos de ASEPUMA, 9, 101-122.

Canós, L., \& Liern, V. (2004). Some fuzzy models for human resources management. International Journal of Technology, Policy and Management, 4(4), 291-308.

Canós, L., \& Liern, V. (2008). Soft computing-based aggregation methods for human resource management. European Journal of Operational Research, 189(3), 669-681.

Chen, C. C., \& Lin, M. M. (2014). The effect of applicant impression management tactics on hiring recommendations: Cognitive and affective processes. Applied Psychology, 63(4), 698-724.

Chun, R. (2005). Corporate reputation: Meaning and measurement. International Journal of Management Reviews, 7(2), 91-109.

Davies, G., Chun, R., da Silva, V. R., \& Roper, S. (2004). A corporate character scale to assess employee and customer views of organization reputation. Corporate Reputation Review, 7(2), 125-146.

Ellis, A. P. J., West, B. J., Ryan, A. M., \& DeShon, R. P. (2002). The use of impression management tactics in structured interviews: A function of question type? Journal of Applied Psychology, 87(6), 1200-1208.

Fombrun, C. J. (1996). Reputation: Realizing value from the corporate image. Boston, MA: Harvard Business School Press.

Fombrun, C. J., \& Low, J. (2011). The real value of reputation. Communication World, 28(6), 18-22.

Foste, E. A., \& Botero, I. C. (2012). Personal reputation: Effects of upward communication on impressions about new employees. Management Communication Quarterly, 26(1), 48-73.

Fullér, R., Canós, M. J., \& Canós, L. (2012). Transparent fuzzy logic based methods for some human resources problems. Rect@ Revista Electrónica de Comunicaciones y Trabajos de ASEPUMA, 13(1), 27-41.

Goguen, J. A. (1967). L-fuzzy sets. Journal of Mathematical Analysis and Applications, 18(1), 145-174.

Goguen, J. A. (1969). The logic of inexact concepts. Synthese, 19(3), 325-373. 
Guillén, M., \& González, T. F. (2001). The ethical dimensions of managerial leadership: Two illustrative case studies in TQM. Journal of Business Ethics, 34(3), 175-189.

Helm, S. (2005). Designing a formative measure for corporate reputation. Corporate Reputation Review, 8(2), 95-109.

Higgins, C. A., \& Judge, T. A. (2004). The effect of applicant influence tactics on recruiter perceptions of fit and hiring recommendations: A field study. Journal of Applied Psychology, 89(4), 622-632.

Highhouse, S., Brooks, M. E., \& Gregarus, G. (2009). An organizational impression management perspective on the formation of corporate reputations. Journal of Management, 35(6), 14811493.

Jácome, R. (2015). La impression management aplicada a la construcción de reputación personal y en las organizaciones: El caso del Dircom (Tesis doctoral). Recuperada de RiuNet Repositorio Institucional Universitat Politècnica de València. doi:10.4995/Thesis/10251/59524.

Jácome, R., Bañón, A. J., Guillén, M., \& Canós, L. (2013). Influencia de la teoría de impression management en los comportamientos que afectan a la relación entre personas y organizaciones. Working Papers on Operations Management, 4(1), 9-22.

Jones, E. E., \& Pittman, T. S. (1982). Toward a general theory of strategic self-presentation. En J. Suls (Ed.), Psychological perspectives on the self (pp. 231-263). Hillsdale, NJ: Lawrence Erlbaum.

Kacmar, K. M., \& Tucker, R. (2014). The moderating effect of supervisor's behavioral integrity on the relationship between regulatory focus and impression management. Journal of Business Ethics, 1-12.

Kaufmann, A., \& Gil Aluja, J. (1987). Técnicas operativas de gestión para el tratamiento de la incertidumbre. Barcelona, España: Hispano Europea.

Keller, K. L. (1993). Conceptualizing, measuring, and managing customer-based brand equity. Journal of Marketing, 57(1), 1-22. 
Kristof-Brown, A. L. (2000). Perceived applicant fit: Distinguishing between recruiters' perceptions of person-job and personorganization fit. Personnel Psychology, 53(3), 643-671.

Kumar, K., \& Beyerlein, M. (1991). Construction and validation of an instrument for measuring ingratiatory behaviors in organizational settings. Journal of Applied Psychology, 76(5), 619-627.

Larsen, H. L. (2002). Efficient importance weighted aggregation between min and max. 9th Conference on information processing and management of uncertainty in knowledge-based systems. Conferencia llevada a cabo en Annecy, Francia.

Leary, M. R., \& Kowalski, R. M. (1990). Impression management: A literature review and two-component model. Psychological Bulletin, 107(1), 34-47.

Liern, V., \& Canós, L. (2003). Toma de decisiones mediante algoritmos borrosos:aplicación a la viabilidad y reestructuración de plantillas laborales. Revista Europea de Dirección y Economía de la Empresa, 12(2), 127-142.

Mayer, R. C., Davis, J. H., \& Schoorman, F. D. (1995). An integrative model of organizational trust. Management, 20(3), 709-734.

Palanski, M. E., Kahai, S. S., \& Yammarino, F. J. (2011). Team virtues and performance: An examination of transparency, behavioral integrity, and trust. Journal of Business Ethics, 99(2), 201-2161.

Proost, K., Schreurs, B., Witte, K. De, \& Derous, E. (2010). Ingratiation and self-promotion in the selection interview: The effects of using single tactics or a combination of tactics on interviewer judgments. Journal of Applied Social Psychology, 40(9), 2155-2169.

Rosenfeld, P., Giacalone, R. A., \& Riordan, C. A. (1995). Impression management in organizations: Theory, measurement, practice. (V. N. Reinhold, Ed.). New York, NY: Routledge: Lawrence Erlbaum.

Schlenker, B. R. (1980). Impression management: The self-concept, social identity, and interpersonal relations. Monterey, CA: Brooks/Cole Publishing Company.

Schweizer, T. S., \& Wijnberg, N. M. (1999). Transferring reputation to the corporation in different cultures: Individuals, collectives, 
systems and the strategic management of corporate reputation. Corporate Reputation Review, 2(3), 249-266.

Scott, S. G., \& Lane, V. R. (2000). A stakeholder approach to organizational identity. Academy of Management Review, 25(1), 43-62.

Simons, T. (2002). Behavioral integrity: The perceived alignment between managers' words and deeds as a research focus. Organization Science, 13(1), 18-35.

Sung, M., \& Yang, S.-U. (2008). Toward the model of university image: The influence of brand personality, external prestige, and reputation. Journal of Public Relations Research, 20(4), 357376.

Valle Cabrera, R. J. (2003). La gestión estratégica de los recursos humanos. Madrid, España: Pearson Educación.

Walker, K. (2010). A systematic review of the corporate reputation literature: Definition, measurement, and theory. Corporate Reputation Review, 12(4), 357-387.

Walsh, G., \& Beatty, S. E. (2007). Customer-based corporate reputation of a service firm: scale development and validation. Journal of the Academy of Marketing Science, 35(1), 127-143.

Wayne, S. J., \& Kacmar, K. M. (1991). The effects of impression management on the performance appraisal process. Organizational Behavior and Human Decision Processes, 48(1), 70. Zadeh, L. A. (1965). Fuzzy sets. Information and Control, 8(3), 338-353. Zinko, R., Ferris, G. R., Humphrey, S. E., Meyer, C. J., \& Aime, F. (2012). Personal reputation in organizations: Two-study constructive replication and extension of antecedents and consequences. Journal of Occupational and Organizational Psychology, 85(1), 156-180. 